$12-7-2021$

\title{
Non-Parametric Tests for Testing of Scale Parameters
}

\author{
Manish Goyal \\ Panjab University, Chandigarh, manishgoyal33@gmail.com \\ Narinder Kumar \\ Panjab University, Chandigarh, nkumar@pu.ac.in
}

Follow this and additional works at: https://digitalcommons.wayne.edu/jmasm

Part of the Applied Statistics Commons, Social and Behavioral Sciences Commons, and the Statistical Theory Commons

\section{Recommended Citation}

Goyal, Manish and Kumar, Narinder (2021) "Non-Parametric Tests for Testing of Scale Parameters," Journal of Modern Applied Statistical Methods: Vol. 19 : Iss. 1 , Article 23.

DOI: $10.22237 / \mathrm{jmasm} / 1619482440$

Available at: https://digitalcommons.wayne.edu/jmasm/vol19/iss1/23

This Regular Article is brought to you for free and open access by the Open Access Journals at DigitalCommons@WayneState. It has been accepted for inclusion in Journal of Modern Applied Statistical Methods by an authorized editor of DigitalCommons@WayneState. 


\section{Non-Parametric Tests for Testing of Scale Parameters}

\section{Cover Page Footnote}

The authors thank the Editor-in-Chief and two anonymous referees for their valuable suggestions, which led to an improvement of the earlier version of the paper. 


\section{Non-Parametric Tests for Testing of Scale Parameters}

\author{
Manish Goyal \\ Panjab University \\ Chandigarh, India
}

\author{
Narinder Kumar \\ Panjab University \\ Chandigarh, India
}

One of the fundamental problems in testing of equality of populations is of testing the equality of scale parameters. The subsequent usages for scale are dispersion, spread and variability. In this paper, we proposed non-parametric tests based on U-Statistics for the testing of equality of scale parameters. The null distribution of proposed tests is developed and its Pitman efficiency is worked out to compare proposed tests with respect to some existing tests. Simulation study is carried out to compute the asymptotic power of proposed tests. An illustrative example is also provided.

Keywords: Two-sample scale, common quantile, null distribution, Pitman efficiency, illustrative example, asymptotic power

\section{Introduction}

Let $X_{1}, \ldots, X_{n_{1}}$ and $Y_{1}, \ldots, Y_{n_{2}}$ be independent random samples from two populations $X$ and $Y$ having absolutely continuous cumulative distribution functions (cdfs) $\mathrm{F}(x)$ and $\mathrm{G}(y)$, respectively. We assumed that both of these distributions are alike except differing in their scale parameters with common known quantile $\xi_{q}$ of order $q, 0 \leq q \leq 1$. Thus, if we take $\mathrm{G}(y)=\mathrm{F}(y / \theta)$, then we wish to test the null hypothesis

$$
\mathrm{H}_{0}: \theta=1, \text { with } \mathrm{F}\left(\xi_{q}\right)=\mathrm{G}\left(\xi_{q}\right)=q
$$

against the alternative hypothesis

$$
\mathrm{H}_{\mathrm{A}}: \theta>1 \text {, with } \mathrm{F}\left(\xi_{q}\right)=\mathrm{G}\left(\xi_{q}\right)=q \text {. }
$$

doi: 10.22237/jmasm/1619482440 | Accepted: January 4, 2019; Published: December 7, 2021.

Correspondence: Narinder Kumar, nkumar@pu.ac.in 


\section{GOYAL \& KUMAR}

Under the null hypothesis, $X \mathrm{~s}$ and $Y \mathrm{~s}$ are alike, but under alternative, $Y \mathrm{~s}$ will have more variation than $X$ s. Without loss of generality, we assume that $\xi_{q}$ is zero for pre-specified $q$.

If both the distributions have common quantile of order 0.5 , then a number of non-parametric tests are available in literature including Mood (1954), Sukhatme (1957), Ansari and Bradley (1960), Klotz (1962), Tamura (1966), Yanagawa (1970), Kochar and Gupta (1986), Kössler (1994, 1999), Öztürk (2001), Kössler and Kumar (2010), and Kumar and Goyal (2018).

Deshpande and Kusum (1984) proposed a test under the assumption that the two distributions have a common known quantile of order $q, 0 \leq q \leq 1$, which was further modified by Mahajan et al. (2011), Kössler and Kumar (2016), and Goyal and Kumar (2018). This type of problem has a number of practical applications in agriculture, engineering, business, industries, biology, atmospheric and chemical sciences.

\section{The Proposed Tests}

Consider $m$ as a fixed non-negative integer and $k$ as a fixed positive integer such that $1 \leq 2 m+1 \leq n_{1}$ and $1 \leq k \leq n_{2}$. Define the following two symmetrical kernels, $h^{(1)}$ and $h^{(2)}$, as

$$
\begin{aligned}
& h^{(1)}\left(X_{1}, \ldots, X_{2 m+1} ; Y_{1}, \ldots, Y_{k}\right)= \begin{cases}1 & \text { if } 0 \leq \operatorname{Med}_{X} \leq \operatorname{Min}_{Y} \text { and } \operatorname{Min}_{X} \geq 0 \\
0 & \text { or } \operatorname{Max}_{Y} \leq \operatorname{Med}_{X} \leq 0 \text { and } \operatorname{Max}_{X} \leq 0 \\
0 \text { otherwise }\end{cases} \\
& h^{(2)}\left(X_{1}, \ldots, X_{2 m+1} ; Y_{1}, \ldots, Y_{k}\right)= \begin{cases}1 & \text { if } 0 \leq \operatorname{Med}_{X} \leq \operatorname{Min}_{Y} \text { and } \operatorname{Min}_{X} \geq 0 \\
& {\text { or } \operatorname{Max}_{Y} \leq \operatorname{Med}_{X} \leq 0 \text { and } \operatorname{Max}_{X} \leq 0} \text { if } 0 \leq \operatorname{Min}_{Y} \leq \operatorname{Med}_{X} \text { and } \operatorname{Min}_{X} \geq 0 \\
-1 & \text { or } \operatorname{Med}_{X} \leq \operatorname{Max}_{Y} \leq 0 \text { and } \operatorname{Max}_{X} \leq 0 \\
0 & \text { otherwise }\end{cases}
\end{aligned}
$$

where $\operatorname{Med}_{X}=$ Median of $X_{1}, \ldots, X_{2 m+1}, \operatorname{Min}_{X}\left(\right.$ or $\left.\operatorname{Max}_{X}\right)=$ Minimum (or Maximum) of $X_{1}, \ldots, X_{2 m+1}$, and $\operatorname{Min}_{Y}\left(\right.$ or $\left.\operatorname{Max}_{Y}\right)=$ Minimum (or Maximum) of $Y_{1}, \ldots, Y_{k}$.

The $U$-Statistics associated with kernel $h^{(c)}, c=1,2$, and of degree $(2 m+1, k)$, is defined as 


\section{TESTING OF SCALE PARAMETERS}

$$
\begin{aligned}
& U_{m, k}^{(c)}\left(X_{1}, \ldots, X_{n_{1}} ; Y_{1}, \ldots, Y_{n_{2}}\right)= \\
& \left.\qquad\left(\begin{array}{c}
n_{1} \\
2 m+1
\end{array}\right)\left(\begin{array}{c}
n_{2} \\
k
\end{array}\right)\right]^{-1} \sum_{s} h^{(c)}\left(X_{i_{1}}, \ldots, X_{i_{m+1}} ; Y_{j_{1}}, \ldots, Y_{j_{k}}\right)
\end{aligned}
$$

where $s$ is summation extended over all possible combinations $\left(i_{1}, \ldots, i_{2 m+1}\right)$ of $2 m+1$ integers chosen from $\left(1, \ldots, n_{1}\right)$ and all possible combinations $\left(j_{1}, \ldots, j_{k}\right)$ of $k$ integers chosen from $\left(1, \ldots, n_{2}\right)$.

The test rejects $\mathrm{H}_{0}$ in favor of $\mathrm{H}_{\mathrm{A}}$ for large values of $U_{m, k}^{(c)} ; c=1$, 2. In particular,

1) For $m=0$ and $k=1$, the test statistic $U_{m, k}^{(1)}$ corresponds to test statistics proposed by Sukhatme (1957).

2) For $m=0$ and $k=1$, the test statistic $U_{m, k}^{(2)}$ corresponds to test statistics proposed by Deshpande and Kusum (1984).

3) For $k=1$, the test statistic $U_{m, k}^{(c)} ; c=1,2$. corresponds to test statistics proposed by Goyal and Kumar (2018).

\section{Distribution of the Test Statistics}

The expectation of test statistic $U_{m, k}^{(c)}$ is

$$
\begin{aligned}
\mathrm{E}\left(U_{m, k}^{(c)}\right) & =\left[\left(\begin{array}{c}
n_{1} \\
2 m+1
\end{array}\right)\left(\begin{array}{c}
n_{2} \\
k
\end{array}\right)\right]^{-1} \sum_{s} h^{(c)}\left(X_{i_{1}}, \ldots, X_{i_{2 m+1}} ; Y_{j_{1}}, \ldots, Y_{j_{k}}\right) \\
& =\mathrm{E}\left[h^{(c)}\left(X_{1}, \ldots, X_{2 m+1} ; Y_{1}, \ldots, Y_{k}\right)\right]
\end{aligned}
$$

For $c=1$,

$$
\mathrm{E}\left(U_{m, k}^{(1)}\right)=\int_{0}^{\infty} \mathrm{P}\left(\operatorname{Med}_{X} \leq t\right) \mathrm{P}\left(\operatorname{Min}_{Y}=t\right) d t+\int_{-\infty}^{0} \mathrm{P}\left(\operatorname{Med}_{X} \geq t\right) \mathrm{P}\left(\operatorname{Max}_{Y}=t\right) d t .
$$

Under $\mathrm{H}_{0}$, 


\section{GOYAL \& KUMAR}

$$
\mathrm{E}\left(U_{m, k}^{(1)}\right)=\frac{k\left[(1-q)^{2 m+k+1}+q^{2 m+k+1}\right]}{(2 m+k+1)}\left\{\sum_{i=m+1}^{2 m+1} \frac{\left(\begin{array}{c}
2 m+1 \\
i
\end{array}\right)}{\left(\begin{array}{c}
2 m+k \\
i
\end{array}\right)}\right\} .
$$

For $c=2$,

$$
\begin{aligned}
\mathrm{E}\left(U_{m, k}^{(2)}\right)=\int_{0}^{\infty} \mathrm{P}\left(\operatorname{Med}_{X} \leq t\right) \mathrm{P}\left(\operatorname{Min}_{Y}=t\right) d t & +\int_{-\infty}^{0} \mathrm{P}\left(\operatorname{Med}_{X} \geq t\right) \mathrm{P}\left(\operatorname{Max}_{Y}=t\right) d t \\
& -\int_{0}^{\infty} \mathrm{P}\left(\operatorname{Med}_{X} \geq t\right) \mathrm{P}\left(\operatorname{Min}_{Y}=t\right) d t-\int_{-\infty}^{0} \mathrm{P}\left(\operatorname{Med}_{X} \leq t\right) \mathrm{P}\left(\operatorname{Max}_{Y}=t\right) d t
\end{aligned}
$$

Under $\mathrm{H}_{0}$,

$$
\mathrm{E}\left(U_{m, k}^{(2)}\right)=\frac{k\left[(1-q)^{2 m+k+1}+q^{2 m+k+1}\right]}{(2 m+k+1)} \sum_{i=m+1}^{2 m+1}\left(\begin{array}{c}
2 m+1 \\
i
\end{array}\right)\left[\frac{1}{\left(\begin{array}{c}
2 m+k \\
i
\end{array}\right)}-\frac{1}{\left(\begin{array}{c}
2 m+k \\
i+k-1
\end{array}\right)}\right] .
$$

The following theorem provides us the asymptotic normality of $U_{m, k}^{(c)}$, which follows from the well-known theory of $U$-Statistics given by Lehmann (1963).

Theorem 1. Let $\mathrm{N}=n_{1}+n_{2}$. The asymptotic distribution of $N^{1 / 2}\left[U_{m, k}^{(c)}-\mathrm{E}\left(U_{m, k}^{(c)}\right)\right]$ as $N \rightarrow \infty$ in such a way that that $\left(n_{1} / N\right) \rightarrow \lambda, 0<\lambda<1$, is normal with mean zero and variance, $\sigma^{2}\left(U_{m, k}^{(c)}\right)$, given by

$$
\sigma^{2}\left(U_{m, k}^{(c)}\right)=\frac{(2 m+1)^{2} \zeta_{10}^{(c)}}{\lambda}+\frac{\zeta_{01}^{(c)}}{1-\lambda}
$$

Here,

$$
\zeta_{10}^{(c)}=\mathrm{E}\left[\left(h^{(c)}\left(x, X_{2}, \ldots, X_{2 m+1} ; Y_{1}, Y_{2}, \ldots, Y_{k}\right)\right)^{2}\right]-\left[\mathrm{E}\left(U_{m, k}^{(c)}\right)\right]^{2}
$$

and 


\section{TESTING OF SCALE PARAMETERS}

$$
\zeta_{01}^{(c)}=\mathrm{E}\left[\left(h^{(c)}\left(X_{1}, X_{2}, \ldots, X_{2 m+1} ; y, Y_{2}, \ldots, Y_{k}\right)\right)^{2}\right]-\left[\mathrm{E}\left(U_{m, k}^{(c)}\right)\right]^{2}
$$

where

$$
h^{(c)}\left(x, X_{2}, \ldots, X_{2 m+1} ; Y_{1}, Y_{2}, \ldots, Y_{k}\right)=\mathrm{E}\left[h^{(c)}\left(X_{1}, X_{2}, \ldots, X_{2 m+1} ; Y_{1}, Y_{2}, \ldots, Y_{k}\right) \mid X_{1}=x\right]
$$

and

$$
h^{(c)}\left(X_{1}, X_{2}, \ldots, X_{2 m+1} ; y, Y_{2}, \ldots, Y_{k}\right)=\mathrm{E}\left[h^{(c)}\left(X_{1}, X_{2}, \ldots, X_{2 m+1} ; Y_{1}, Y_{2}, \ldots, Y_{k}\right) \mid Y_{1}=y\right]
$$

Under $\mathrm{H}_{0}$, after some complex but standard computations, we elaborate the asymptotic null variance, $\sigma_{0}^{2}\left(U_{m, k}^{(c)}\right)$, as

$$
\sigma_{0}^{2}\left(U_{m, k}^{(c)}\right)=\frac{(2 m+1)^{2}\left\{k^{2} \rho_{m, k}^{(c)}\left[(1-q)^{4 m+2 k+1}+q^{4 m+2 k+1}\right]-\left[\mathrm{E}\left(U_{m, k}^{(c)}\right)\right]^{2}\right\}}{\lambda(1-\lambda)}
$$

where, for $c=1$,

$$
\begin{aligned}
& \rho_{m, k}^{(1)}=\left(\begin{array}{c}
2 m \\
m
\end{array}\right)^{2} \sum_{i=0}^{m} \sum_{j=0}^{m}\left(\begin{array}{c}
m \\
i
\end{array}\right)\left(\begin{array}{c}
m \\
j
\end{array}\right) \frac{(-1)^{i+j}(4 m+2 k-i-j+1)^{-1}}{(2 m+k-i)(2 m+k-j)} \\
& +\frac{2\left(\begin{array}{c}
2 m \\
m
\end{array}\right)}{(2 m+k)}\left[\sum_{i=m+1}^{2 m} \frac{\left(\begin{array}{c}
2 m \\
i
\end{array}\right)}{\left(\begin{array}{c}
2 m+k-1 \\
i
\end{array}\right)}\right]\left[\sum_{j=0}^{m}\left(\begin{array}{c}
m \\
j
\end{array}\right) \frac{(-1)^{m+j}(2 m+k-j+1)^{-1}}{(2 m+k-j)}\right] \\
& +\frac{1}{(2 m+k)^{2}}\left[\sum_{i=m+1}^{2 m} \frac{\left(\begin{array}{c}
2 m \\
i
\end{array}\right)}{\left(\begin{array}{c}
2 m+k-1 \\
i
\end{array}\right)}\right]^{2}
\end{aligned}
$$

and for $c=2$, 


$$
\begin{aligned}
& \rho_{m, k}^{(2)}=4\left(\begin{array}{c}
2 m \\
m
\end{array}\right)^{2} \sum_{i=0}^{m} \sum_{j=0}^{m}\left(\begin{array}{c}
m \\
i
\end{array}\right)\left(\begin{array}{c}
m \\
j
\end{array}\right) \frac{(-1)^{i+j}(4 m+2 k-i-j+1)^{-1}}{(2 m+k-i)(2 m+k-j)} \\
& +\frac{4\left(\begin{array}{c}
2 m \\
m
\end{array}\right)}{(2 m+k)}\left[\sum_{i=m+1}^{2 m} \frac{\left(\begin{array}{c}
2 m \\
i
\end{array}\right)}{\left(\begin{array}{c}
2 m+k-1 \\
i
\end{array}\right)}-\sum_{i=m}^{2 m} \frac{\left(\begin{array}{c}
2 m \\
i
\end{array}\right)}{\left(\begin{array}{c}
2 m+k-1 \\
i+k-1
\end{array}\right)}\right]\left[\sum_{j=0}^{m}\left(\begin{array}{c}
m \\
j
\end{array}\right) \frac{(-1)^{m+j}(2 m+k-j+1)^{-1}}{(2 m+k-j)}\right] \\
& +\frac{1}{(2 m+k)^{2}}\left[\sum_{i=m+1}^{2 m} \frac{\left(\begin{array}{c}
2 m \\
i
\end{array}\right)}{\left(\begin{array}{c}
2 m+k-1 \\
i
\end{array}\right)}-\sum_{i=m}^{2 m} \frac{\left(\begin{array}{c}
2 m \\
i
\end{array}\right)}{\left(\begin{array}{c}
2 m+k-1 \\
i+k-1
\end{array}\right)}\right]^{2}
\end{aligned}
$$

\section{Asymptotic Relative Efficiency}

In order to see the performance of the proposed tests with respect to existing tests, we compute the Pitman asymptotic relative efficiencies (AREs) of $U_{m, k}^{(c)}$ tests relative to some existing tests, namely Sukhatme (1957) test $(S)$, Mahajan et al. (2011) test $(M G A)$, and some members of Kössler and Kumar (2016) test $\left(T_{l}\right)$. Moreover, we also compare the proposed tests $U_{m, k}^{(1)}$ and $U_{m, k}^{(2)}$ with each other as well.

Remark 1. Pitman AREs of the proposed tests with respect to Deshpande and Kusum (1984) test is same as that of AREs with respect to Kössler and Kumar (2016) test $\left(T_{l}\right)$ for $l=1$.

The efficacy of the test statistics $U_{m, k}^{(c)}$ under the sequence of local alternatives, $\theta_{N}=N^{(-1 / 2)} \theta$, is 


\section{TESTING OF SCALE PARAMETERS}

$$
\begin{aligned}
& \mathrm{e}^{2}\left(U_{m, k}^{(c)}\right)=\lim _{N \rightarrow \infty} \frac{\left[\left.\frac{d}{d \theta} E\left(U_{m, k}^{(c)}\right)\right|_{\theta=1}\right]^{2}}{N \sigma_{0}^{2}\left(U_{m, k}^{(c)}\right)} \\
&=\frac{c^{2}\left[(2 m+1)\left(\begin{array}{c}
2 m \\
m
\end{array}\right) k\right]^{2}}{\sigma_{0}^{2}\left(U_{m, k}^{(c)}\right)}\left[\int_{0}^{\infty}(\mathrm{F}(y)-q)^{m}(1-\mathrm{F}(y))^{m+k-1} \mathrm{f}^{2}(y) d y\right. \\
&\left.-\int_{-\infty}^{0}(\mathrm{~F}(y))^{m+k-1}(q-\mathrm{F}(y))^{m} \mathrm{f}^{2}(y) d y\right]^{2}
\end{aligned}
$$

The efficacies of the $S, M G A$, and $T_{l}$ tests are

$$
\begin{aligned}
\mathrm{e}^{2}(S)= & \frac{\lambda(1-\lambda)}{\frac{1}{12}-q^{2}(1-q)^{2}}\left\{\int_{-\infty}^{\infty}|y| \mathrm{f}^{2}(y) d y\right\}^{2} \\
\mathrm{e}^{2}(M G A)= & \frac{831600 \lambda(1-\lambda)}{131\left(q^{11}+(1-q)^{11}\right)}\left\{\int_{-\infty}^{0} y \mathrm{~F}^{2}(y)[q-\mathrm{F}(y)]^{2} \mathrm{f}^{2}(y) d y\right. \\
& \left.-\int_{0}^{\infty} y[\mathrm{~F}(y)-q]^{2}[1-\mathrm{F}(y)]^{2} \mathrm{f}^{2}(y) d y\right\}^{2} \\
\mathrm{e}^{2}\left(T_{l}\right)= & \frac{4 l^{2}(4 l-1) \lambda(1-\lambda)}{\left(q^{4 l-1}+(1-q)^{4 l-1}\right)}\left\{\int_{-\infty}^{\infty}|y|[\mathrm{F}(y)-q]^{2 l-2} \mathrm{f}^{2}(y) d y\right\}^{2}
\end{aligned}
$$

AREs of $U_{m, k}^{(1)}$ and $U_{m, k}^{(2)}$ tests with respect to (w.r.t.) competing tests for some underlying distributions are given in Tables 1-10. 


\section{GOYAL \& KUMAR}

Table 1. AREs of $U_{m, k}^{(1)}$ w.r.t. competing tests for Uniform distribution

\begin{tabular}{|c|c|c|c|c|c|c|c|c|c|c|}
\hline \multirow[b]{3}{*}{ Test } & \multicolumn{10}{|c|}{$q$ or $(1-q)$} \\
\hline & \multirow[b]{2}{*}{$m$} & \multicolumn{3}{|c|}{0.1} & \multicolumn{3}{|c|}{0.3} & \multicolumn{3}{|c|}{0.5} \\
\hline & & $k=1$ & $k=2$ & $k=3$ & $k=1$ & $k=2$ & $k=3$ & $k=1$ & $k=2$ & $k=3$ \\
\hline \multirow[t]{3}{*}{$S$} & 0 & 1.0000 & 0.4500 & 0.2908 & 1.0000 & 0.3757 & 0.2260 & 1.0000 & 0.4167 & 0.2593 \\
\hline & 1 & 0.2913 & 0.3118 & 0.2702 & 0.1256 & 0.1525 & 0.1465 & 0.6863 & 0.4242 & 0.3056 \\
\hline & 2 & 0.1313 & 0.2119 & 0.2256 & 0.0421 & 0.0804 & 0.0987 & 0.5878 & 0.4222 & 0.3287 \\
\hline \multirow[t]{3}{*}{$M G A$} & 0 & 1.4079 & 0.6336 & 0.4095 & 1.7154 & 0.6445 & 0.3878 & 1.7013 & 0.7089 & 0.4411 \\
\hline & 1 & 0.4101 & 0.4390 & 0.3804 & 0.2154 & 0.2616 & 0.2513 & 1.1676 & 0.7218 & 0.5198 \\
\hline & 2 & 0.1849 & 0.2984 & 0.3176 & 0.0722 & 0.1380 & 0.1693 & 1.0000 & 0.7184 & 0.5593 \\
\hline \multirow[t]{3}{*}{$T_{1}$} & 0 & 0.8086 & 0.3639 & 0.2352 & 0.7859 & 0.2952 & 0.1776 & 1.0000 & 0.4167 & 0.2593 \\
\hline & 1 & 0.2355 & 0.2521 & 0.2185 & 0.0987 & 0.1198 & 0.1151 & 0.6863 & 0.4242 & 0.3056 \\
\hline & 2 & 0.1062 & 0.1714 & 0.1824 & 0.0331 & 0.0632 & 0.0776 & 0.5878 & 0.4222 & 0.3287 \\
\hline \multirow[t]{3}{*}{$T_{2}$} & 0 & 0.3546 & 0.1595 & 0.1031 & 0.4105 & 0.1542 & 0.0928 & 0.4286 & 0.1786 & 0.1111 \\
\hline & 1 & 0.1033 & 0.1106 & 0.0958 & 0.0515 & 0.0626 & 0.0601 & 0.2941 & 0.1818 & 0.1310 \\
\hline & 2 & 0.0466 & 0.0751 & 0.0800 & 0.0173 & 0.0330 & 0.0405 & 0.2519 & 0.1810 & 0.1409 \\
\hline \multirow[t]{3}{*}{$T_{3}$} & 0 & 0.2257 & 0.1016 & 0.0656 & 0.2750 & 0.1033 & 0.0622 & 0.2727 & 0.1136 & 0.0707 \\
\hline & 1 & 0.0657 & 0.0704 & 0.0610 & 0.0345 & 0.0419 & 0.0403 & 0.1872 & 0.1157 & 0.0833 \\
\hline & 2 & 0.0296 & 0.0478 & 0.0509 & 0.0116 & 0.0221 & 0.0271 & 0.1603 & 0.1152 & 0.0897 \\
\hline \multirow{3}{*}{$U_{m, k}^{(2)}$} & 0 & 0.8086 & 0.9185 & 1.0000 & 0.7859 & 0.8518 & 1.0000 & 1.0000 & 1.0000 & 1.0000 \\
\hline & 1 & 0.3511 & 0.7823 & 1.3354 & 0.1753 & 0.6426 & 1.6456 & 1.0000 & 1.0000 & 1.0000 \\
\hline & 2 & 0.1849 & 0.7442 & 1.9680 & 0.0722 & 0.6471 & 2.5552 & 1.0000 & 1.0000 & 1.0000 \\
\hline
\end{tabular}

Table 2. AREs of $U_{m, k}^{(1)}$ w.r.t. competing tests for Normal distribution

\begin{tabular}{|c|c|c|c|c|c|c|c|c|c|c|}
\hline \multirow[b]{3}{*}{ Test } & \multirow[b]{3}{*}{$m$} & \multicolumn{9}{|c|}{$q$ or $(1-q)$} \\
\hline & & \multicolumn{3}{|c|}{0.1} & \multicolumn{3}{|c|}{0.3} & \multicolumn{3}{|c|}{0.5} \\
\hline & & $k=1$ & $k=2$ & $k=3$ & $k=1$ & $k=2$ & $k=3$ & $k=1$ & $k=2$ & $k=3$ \\
\hline \multirow[t]{3}{*}{$S$} & 0 & 1.0000 & 0.7179 & 0.5370 & 1.0000 & 0.6122 & 0.4356 & 1.0000 & 0.6699 & 0.4946 \\
\hline & 1 & 0.3977 & 0.5278 & 0.5059 & 0.1752 & 0.2650 & 0.2836 & 0.9150 & 0.7088 & 0.5739 \\
\hline & 2 & 0.1999 & 0.3696 & 0.4251 & 0.0657 & 0.1437 & 0.1911 & 0.8672 & 0.7196 & 0.6123 \\
\hline \multirow[t]{3}{*}{$M G A$} & 0 & 0.9251 & 0.6642 & 0.4968 & 1.0998 & 0.6733 & 0.4791 & 1.1531 & 0.7724 & 0.5703 \\
\hline & 1 & 0.3679 & 0.4883 & 0.4680 & 0.1927 & 0.2915 & 0.3119 & 1.0551 & 0.8173 & 0.6617 \\
\hline & 2 & 0.1849 & 0.3420 & 0.3933 & 0.0722 & 0.1581 & 0.2101 & 1.0000 & 0.8298 & 0.7060 \\
\hline \multirow[t]{3}{*}{$T_{1}$} & 0 & 0.8086 & 0.5805 & 0.4343 & 0.7859 & 0.4811 & 0.3423 & 1.0000 & 0.6699 & 0.4946 \\
\hline & 1 & 0.3216 & 0.4268 & 0.4091 & 0.1377 & 0.2083 & 0.2229 & 0.9150 & 0.7088 & 0.5739 \\
\hline & 2 & 0.1616 & 0.2989 & 0.3438 & 0.0516 & 0.1130 & 0.1502 & 0.8672 & 0.7196 & 0.6123 \\
\hline \multirow[t]{3}{*}{$T_{2}$} & 0 & 0.5866 & 0.4212 & 0.3151 & 0.6525 & 0.3995 & 0.2842 & 0.7232 & 0.4845 & 0.3577 \\
\hline & 1 & 0.2333 & 0.3096 & 0.2968 & 0.1143 & 0.1729 & 0.1851 & 0.6618 & 0.5126 & 0.4150 \\
\hline & 2 & 0.1173 & 0.2168 & 0.2494 & 0.0429 & 0.0938 & 0.1247 & 0.6272 & 0.5204 & 0.4428 \\
\hline \multirow[t]{3}{*}{$T_{3}$} & 0 & 0.5485 & 0.3938 & 0.2946 & 0.6273 & 0.3840 & 0.2732 & 0.6690 & 0.4481 & 0.3309 \\
\hline & 1 & 0.2181 & 0.2895 & 0.2775 & 0.1099 & 0.1662 & 0.1779 & 0.6122 & 0.4742 & 0.3839 \\
\hline & 2 & 0.1096 & 0.2027 & 0.2332 & 0.0412 & 0.0902 & 0.1199 & 0.5802 & 0.4814 & 0.4096 \\
\hline \multirow{3}{*}{$U_{m, k}^{(2)}$} & 0 & 0.8086 & 0.9185 & 1.0000 & 0.7859 & 0.8518 & 1.0000 & 1.0000 & 1.0000 & 1.0000 \\
\hline & 1 & 0.3511 & 0.7823 & 1.3354 & 0.1753 & 0.6426 & 1.6456 & 1.0000 & 1.0000 & 1.0000 \\
\hline & 2 & 0.1849 & 0.7442 & 1.9680 & 0.0722 & 0.6471 & 2.5552 & 1.0000 & 1.0000 & 1.0000 \\
\hline
\end{tabular}




\section{TESTING OF SCALE PARAMETERS}

Table 3. AREs of $U_{m, k}^{(1)}$ w.r.t. competing tests for Logistic distribution

\begin{tabular}{|c|c|c|c|c|c|c|c|c|c|c|}
\hline \multirow[b]{3}{*}{ Test } & \multirow[b]{3}{*}{$m$} & \multicolumn{9}{|c|}{$q$ or $(1-q)$} \\
\hline & & \multicolumn{3}{|c|}{0.1} & \multicolumn{3}{|c|}{0.3} & \multicolumn{3}{|c|}{0.5} \\
\hline & & $k=1$ & $k=2$ & $k=3$ & $k=1$ & $k=2$ & $k=3$ & $k=1$ & $k=2$ & $k=3$ \\
\hline \multirow[t]{3}{*}{$S$} & 0 & 1.0000 & 0.7685 & 0.5900 & 1.0000 & 0.6552 & 0.4809 & 1.0000 & 0.7124 & 0.5419 \\
\hline & 1 & 0.4129 & 0.5705 & 0.5581 & 0.1816 & 0.2859 & 0.3129 & 0.9385 & 0.7549 & 0.6251 \\
\hline & 2 & 0.2112 & 0.4018 & 0.4698 & 0.0692 & 0.1557 & 0.2105 & 0.9002 & 0.7673 & 0.6647 \\
\hline \multirow[t]{3}{*}{$M G A$} & 0 & 0.8756 & 0.6728 & 0.5166 & 1.0441 & 0.6841 & 0.5021 & 1.1109 & 0.7914 & 0.6020 \\
\hline & 1 & 0.3615 & 0.4995 & 0.4886 & 0.1897 & 0.2986 & 0.3267 & 1.0426 & 0.8386 & 0.6944 \\
\hline & 2 & 0.1849 & 0.3518 & 0.4114 & 0.0722 & 0.1625 & 0.2198 & 1.0000 & 0.8524 & 0.7384 \\
\hline \multirow[t]{3}{*}{$T_{1}$} & 0 & 0.8086 & 0.6214 & 0.4771 & 0.7859 & 0.5149 & 0.3779 & 1.0000 & 0.7124 & 0.5419 \\
\hline & 1 & 0.3339 & 0.4613 & 0.4512 & 0.1427 & 0.2247 & 0.2459 & 0.9385 & 0.7549 & 0.6251 \\
\hline & 2 & 0.1708 & 0.3249 & 0.3799 & 0.0544 & 0.1223 & 0.1655 & 0.9002 & 0.7673 & 0.6647 \\
\hline \multirow[t]{3}{*}{$T_{2}$} & 0 & 0.6438 & 0.4948 & 0.3799 & 0.7073 & 0.4635 & 0.3401 & 0.7858 & 0.5598 & 0.4258 \\
\hline & 1 & 0.2658 & 0.3673 & 0.3593 & 0.1285 & 0.2023 & 0.2213 & 0.7375 & 0.5932 & 0.4912 \\
\hline & 2 & 0.1360 & 0.2587 & 0.3025 & 0.0489 & 0.1101 & 0.1489 & 0.7074 & 0.6030 & 0.5224 \\
\hline \multirow[t]{3}{*}{$T_{3}$} & 0 & 0.6475 & 0.4976 & 0.3821 & 0.7229 & 0.4737 & 0.3476 & 0.7687 & 0.5476 & 0.4166 \\
\hline & 1 & 0.2674 & 0.3694 & 0.3614 & 0.1313 & 0.2067 & 0.2262 & 0.7215 & 0.5803 & 0.4805 \\
\hline & 2 & 0.1367 & 0.2602 & 0.3042 & 0.0500 & 0.1125 & 0.1522 & 0.6920 & 0.5898 & 0.5110 \\
\hline \multirow{3}{*}{$U_{m, k}^{(2)}$} & 0 & 0.8086 & 0.9185 & 1.0000 & 0.7859 & 0.8518 & 1.0000 & 1.0000 & 1.0000 & 1.0000 \\
\hline & 1 & 0.3511 & 0.7823 & 1.3354 & 0.1753 & 0.6426 & 1.6456 & 1.0000 & 1.0000 & 1.0000 \\
\hline & 2 & 0.1849 & 0.7442 & 1.9680 & 0.0722 & 0.6471 & 2.5552 & 1.0000 & 1.0000 & 1.0000 \\
\hline
\end{tabular}

Table 4. AREs of $U_{m, k}^{(1)}$ w.r.t. competing tests for Laplace distribution

\begin{tabular}{|c|c|c|c|c|c|c|c|c|c|c|}
\hline \multirow[b]{3}{*}{ Test } & \multirow[b]{3}{*}{$m$} & \multicolumn{9}{|c|}{$q$ or $(1-q)$} \\
\hline & & \multicolumn{3}{|c|}{0.1} & \multicolumn{3}{|c|}{0.3} & \multicolumn{3}{|c|}{0.5} \\
\hline & & $k=1$ & $k=2$ & $k=3$ & $k=1$ & $k=2$ & $k=3$ & $k=1$ & $k=2$ & $k=3$ \\
\hline \multirow[t]{3}{*}{$S$} & 0 & 1.0000 & 0.8791 & 0.7105 & 1.0000 & 0.7425 & 0.5982 & 1.0000 & 0.7407 & 0.5833 \\
\hline & 1 & 0.4436 & 0.6786 & 0.6972 & 0.1839 & 0.3212 & 0.3817 & 0.9341 & 0.7732 & 0.6573 \\
\hline & 2 & 0.2372 & 0.4908 & 0.6011 & 0.0705 & 0.1730 & 0.2518 & 0.8941 & 0.7795 & 0.6897 \\
\hline \multirow[t]{3}{*}{$M G A$} & 0 & 0.7794 & 0.6852 & 0.5538 & 1.0247 & 0.7609 & 0.6130 & 1.1185 & 0.8285 & 0.6524 \\
\hline & 1 & 0.3458 & 0.5289 & 0.5434 & 0.1884 & 0.3291 & 0.3912 & 1.0447 & 0.8648 & 0.7351 \\
\hline & 2 & 0.1849 & 0.3825 & 0.4685 & 0.0722 & 0.1773 & 0.2581 & 1.0000 & 0.8719 & 0.7713 \\
\hline \multirow[t]{3}{*}{$T_{1}$} & 0 & 0.8086 & 0.7108 & 0.5745 & 0.7859 & 0.5835 & 0.4701 & 1.0000 & 0.7407 & 0.5833 \\
\hline & 1 & 0.3587 & 0.5487 & 0.5637 & 0.1445 & 0.2524 & 0.3000 & 0.9341 & 0.7732 & 0.6573 \\
\hline & 2 & 0.1918 & 0.3968 & 0.4860 & 0.0554 & 0.1360 & 0.1979 & 0.8941 & 0.7795 & 0.6897 \\
\hline \multirow[t]{3}{*}{$T_{2}$} & 0 & 0.7937 & 0.6978 & 0.5640 & 0.8433 & 0.6262 & 0.5045 & 0.8216 & 0.6086 & 0.4793 \\
\hline & 1 & 0.3521 & 0.5386 & 0.5534 & 0.1550 & 0.2709 & 0.3219 & 0.7675 & 0.6353 & 0.5401 \\
\hline & 2 & 0.1883 & 0.3895 & 0.4771 & 0.0594 & 0.1459 & 0.2124 & 0.7346 & 0.6405 & 0.5666 \\
\hline \multirow[t]{3}{*}{$T_{3}$} & 0 & 0.9290 & 0.8167 & 0.6601 & 0.9259 & 0.6875 & 0.5539 & 0.8107 & 0.6005 & 0.4729 \\
\hline & 1 & 0.4121 & 0.6304 & 0.6477 & 0.1702 & 0.2974 & 0.3534 & 0.7573 & 0.6268 & 0.5329 \\
\hline & 2 & 0.2204 & 0.4559 & 0.5584 & 0.0653 & 0.1602 & 0.2332 & 0.7249 & 0.6320 & 0.5591 \\
\hline \multirow{3}{*}{$U_{m, k}^{(2)}$} & 0 & 0.8086 & 0.9185 & 1.0000 & 0.7859 & 0.8518 & 1.0000 & 1.0000 & 1.0000 & 1.0000 \\
\hline & 1 & 0.3511 & 0.7823 & 1.3354 & 0.1753 & 0.6426 & 1.6456 & 1.0000 & 1.0000 & 1.0000 \\
\hline & 2 & 0.1849 & 0.7442 & 1.9680 & 0.0722 & 0.6471 & 2.5552 & 1.0000 & 1.0000 & 1.0000 \\
\hline
\end{tabular}




\section{GOYAL \& KUMAR}

Table 5. AREs of $U_{m, k}^{(1)}$ w.r.t. competing tests for Cauchy distribution

\begin{tabular}{|c|c|c|c|c|c|c|c|c|c|c|}
\hline \multirow[b]{3}{*}{ Test } & \multirow[b]{3}{*}{$m$} & \multicolumn{9}{|c|}{$q$ or $(1-q)$} \\
\hline & & \multicolumn{3}{|c|}{0.1} & \multicolumn{3}{|c|}{0.3} & \multicolumn{3}{|c|}{0.5} \\
\hline & & $k=1$ & $k=2$ & $k=3$ & $k=1$ & $k=2$ & $k=3$ & $k=1$ & $k=2$ & $k=3$ \\
\hline \multirow[t]{3}{*}{$S$} & 0 & 1.0000 & 1.0286 & 0.8835 & 1.0000 & 0.8958 & 0.7676 & 1.0000 & 0.9375 & 0.8253 \\
\hline & 1 & 0.4798 & 0.8026 & 0.8643 & 0.2086 & 0.4010 & 0.4940 & 1.0145 & 0.9799 & 0.9117 \\
\hline & 2 & 0.2649 & 0.5818 & 0.7395 & 0.0845 & 0.2203 & 0.3286 & 1.0121 & 0.9897 & 0.9441 \\
\hline \multirow[t]{3}{*}{$M G A$} & 0 & 0.6981 & 0.7180 & 0.6168 & 0.8547 & 0.7656 & 0.6561 & 0.9880 & 0.9262 & 0.8154 \\
\hline & 1 & 0.3349 & 0.5603 & 0.6033 & 0.1783 & 0.3428 & 0.4222 & 1.0023 & 0.9682 & 0.9008 \\
\hline & 2 & 0.1849 & 0.4061 & 0.5162 & 0.0722 & 0.1883 & 0.2808 & 1.0000 & 0.9778 & 0.9328 \\
\hline \multirow[t]{3}{*}{$T_{1}$} & 0 & 0.8086 & 0.8317 & 0.7144 & 0.7859 & 0.7040 & 0.6032 & 1.0000 & 0.9375 & 0.8253 \\
\hline & 1 & 0.3879 & 0.6490 & 0.6989 & 0.1640 & 0.3152 & 0.3882 & 1.0145 & 0.9799 & 0.9117 \\
\hline & 2 & 0.2142 & 0.4705 & 0.5979 & 0.0664 & 0.1731 & 0.2582 & 1.0121 & 0.9897 & 0.9441 \\
\hline \multirow[t]{3}{*}{$T_{2}$} & 0 & 1.0503 & 1.0803 & 0.9280 & 1.0879 & 0.9745 & 0.8351 & 1.2117 & 1.1360 & 1.0000 \\
\hline & 1 & 0.5039 & 0.8430 & 0.9078 & 0.2270 & 0.4363 & 0.5374 & 1.2293 & 1.1874 & 1.1048 \\
\hline & 2 & 0.2782 & 0.6111 & 0.7767 & 0.0919 & 0.2397 & 0.3574 & 1.2264 & 1.1992 & 1.1440 \\
\hline \multirow[t]{3}{*}{$T_{3}$} & 0 & 1.6322 & 1.6788 & 1.4421 & 1.5549 & 1.3929 & 1.1936 & 1.5807 & 1.4819 & 1.3045 \\
\hline & 1 & 0.7831 & 1.3101 & 1.4107 & 0.3244 & 0.6236 & 0.7682 & 1.6037 & 1.5490 & 1.4412 \\
\hline & 2 & 0.4323 & 0.9496 & 1.2069 & 0.1314 & 0.3425 & 0.5109 & 1.5999 & 1.5644 & 1.4924 \\
\hline \multirow{3}{*}{$U_{m, k}^{(2)}$} & 0 & 0.8086 & 0.9185 & 1.0000 & 0.7859 & 0.8518 & 1.0000 & 1.0000 & 1.0000 & 1.0000 \\
\hline & 1 & 0.3511 & 0.7823 & 1.3354 & 0.1753 & 0.6426 & 1.6456 & 1.0000 & 1.0000 & 1.0000 \\
\hline & 2 & 0.1849 & 0.7442 & 1.9680 & 0.0722 & 0.6471 & 2.5552 & 1.0000 & 1.0000 & 1.0000 \\
\hline
\end{tabular}

Table 6. AREs of $U_{m, k}^{(2)}$ w.r.t. competing tests for Uniform distribution

\begin{tabular}{|c|c|c|c|c|c|c|c|c|c|c|}
\hline \multirow[b]{3}{*}{ Test } & \multicolumn{10}{|c|}{$q$ or $(1-q)$} \\
\hline & \multirow[b]{2}{*}{$m$} & \multicolumn{3}{|c|}{0.1} & \multicolumn{3}{|c|}{0.3} & \multicolumn{3}{|c|}{0.5} \\
\hline & & $k=1$ & $k=2$ & $k=3$ & $k=1$ & $k=2$ & $k=3$ & $k=1$ & $k=2$ & $k=3$ \\
\hline \multirow[t]{3}{*}{$S$} & 0 & 1.2367 & 0.4899 & 0.2908 & 1.2724 & 0.4411 & 0.2260 & 1.0000 & 0.4167 & 0.2593 \\
\hline & 1 & 0.8295 & 0.3986 & 0.2023 & 0.7165 & 0.2373 & 0.0890 & 0.6863 & 0.4242 & 0.3056 \\
\hline & 2 & 0.7103 & 0.2848 & 0.1146 & 0.5829 & 0.1243 & 0.0386 & 0.5878 & 0.4222 & 0.3287 \\
\hline \multirow[t]{3}{*}{$M G A$} & 0 & 1.7412 & 0.6898 & 0.4095 & 2.1828 & 0.7566 & 0.3878 & 1.7013 & 0.7089 & 0.4411 \\
\hline & 1 & 1.1679 & 0.5611 & 0.2848 & 1.2292 & 0.4070 & 0.1527 & 1.1676 & 0.7218 & 0.5198 \\
\hline & 2 & 1.0000 & 0.4009 & 0.1614 & 1.0000 & 0.2132 & 0.0663 & 1.0000 & 0.7184 & 0.5593 \\
\hline \multirow[t]{3}{*}{$T_{1}$} & 0 & 1.0000 & 0.3962 & 0.2352 & 1.0000 & 0.3466 & 0.1776 & 1.0000 & 0.4167 & 0.2593 \\
\hline & 1 & 0.6708 & 0.3223 & 0.1636 & 0.5631 & 0.1865 & 0.0700 & 0.6863 & 0.4242 & 0.3056 \\
\hline & 2 & 0.5743 & 0.2303 & 0.0927 & 0.4581 & 0.0977 & 0.0304 & 0.5878 & 0.4222 & 0.3287 \\
\hline \multirow[t]{3}{*}{$T_{2}$} & 0 & 0.4385 & 0.1737 & 0.1031 & 0.5223 & 0.1810 & 0.0928 & 0.4286 & 0.1786 & 0.1111 \\
\hline & 1 & 0.2941 & 0.1413 & 0.0717 & 0.2941 & 0.0974 & 0.0365 & 0.2941 & 0.1818 & 0.1310 \\
\hline & 2 & 0.2518 & 0.1010 & 0.0406 & 0.2393 & 0.0510 & 0.0159 & 0.2519 & 0.1810 & 0.1409 \\
\hline \multirow[t]{3}{*}{$T_{3}$} & 0 & 0.2791 & 0.1106 & 0.0656 & 0.3499 & 0.1213 & 0.0622 & 0.2727 & 0.1136 & 0.0707 \\
\hline & 1 & 0.1872 & 0.0900 & 0.0457 & 0.1970 & 0.0653 & 0.0245 & 0.1872 & 0.1157 & 0.0833 \\
\hline & 2 & 0.1603 & 0.0643 & 0.0259 & 0.1603 & 0.0342 & 0.0106 & 0.1603 & 0.1152 & 0.0897 \\
\hline \multirow{3}{*}{$U_{m, k}^{(1)}$} & 0 & 1.2367 & 1.0887 & 1.0000 & 1.2724 & 1.1740 & 1.0000 & 1.0000 & 1.0000 & 1.0000 \\
\hline & 1 & 2.8479 & 1.2782 & 0.7489 & 5.7056 & 1.5562 & 0.6077 & 1.0000 & 1.0000 & 1.0000 \\
\hline & 2 & 5.4083 & 1.3437 & 0.5081 & 13.8442 & 1.5453 & 0.3914 & 1.0000 & 1.0000 & 1.0000 \\
\hline
\end{tabular}




\section{TESTING OF SCALE PARAMETERS}

Table 7. AREs of $U_{m, k}^{(2)}$ w.r.t. competing tests for Normal distribution

\begin{tabular}{|c|c|c|c|c|c|c|c|c|c|c|}
\hline \multirow[b]{3}{*}{ Test } & & \multicolumn{9}{|c|}{$q$ or $(1-q)$} \\
\hline & \multirow[b]{2}{*}{$m$} & \multicolumn{3}{|c|}{0.1} & \multicolumn{3}{|c|}{0.3} & \multicolumn{3}{|c|}{0.5} \\
\hline & & $k=1$ & $k=2$ & $k=3$ & $k=1$ & $k=2$ & $k=3$ & $k=1$ & $k=2$ & $k=3$ \\
\hline \multirow[t]{3}{*}{$S$} & 0 & 1.2367 & 0.7817 & 0.5370 & 1.2724 & 0.7187 & 0.4356 & 1.0000 & 0.6699 & 0.4946 \\
\hline & 1 & 1.1325 & 0.6746 & 0.3789 & 0.9995 & 0.4124 & 0.1723 & 0.9150 & 0.7088 & 0.5739 \\
\hline & 2 & 1.0810 & 0.4967 & 0.2160 & 0.9092 & 0.2221 & 0.0748 & 0.8672 & 0.7196 & 0.6123 \\
\hline \multirow[t]{3}{*}{$M G A$} & 0 & 1.1441 & 0.7231 & 0.4968 & 1.3995 & 0.7905 & 0.4791 & 1.1531 & 0.7724 & 0.5703 \\
\hline & 1 & 1.0477 & 0.6241 & 0.3505 & 1.0993 & 0.4536 & 0.1896 & 1.0551 & 0.8173 & 0.6617 \\
\hline & 2 & 1.0000 & 0.4595 & 0.1998 & 1.0000 & 0.2443 & 0.0822 & 1.0000 & 0.8298 & 0.7060 \\
\hline \multirow[t]{3}{*}{$T_{1}$} & 0 & 1.0000 & 0.6320 & 0.4343 & 1.0000 & 0.5648 & 0.3423 & 1.0000 & 0.6699 & 0.4946 \\
\hline & 1 & 0.9157 & 0.5455 & 0.3064 & 0.7855 & 0.3241 & 0.1354 & 0.9150 & 0.7088 & 0.5739 \\
\hline & 2 & 0.8741 & 0.4016 & 0.1747 & 0.7146 & 0.1746 & 0.0588 & 0.8672 & 0.7196 & 0.6123 \\
\hline \multirow[t]{3}{*}{$T_{2}$} & 0 & 0.7255 & 0.4586 & 0.3151 & 0.8303 & 0.4690 & 0.2842 & 0.7232 & 0.4845 & 0.3577 \\
\hline & 1 & 0.6644 & 0.3958 & 0.2223 & 0.6522 & 0.2691 & 0.1125 & 0.6618 & 0.5126 & 0.4150 \\
\hline & 2 & 0.6341 & 0.2914 & 0.1267 & 0.5933 & 0.1450 & 0.0488 & 0.6272 & 0.5204 & 0.4428 \\
\hline \multirow[t]{3}{*}{$T_{3}$} & 0 & 0.6783 & 0.4287 & 0.2946 & 0.7982 & 0.4508 & 0.2732 & 0.6690 & 0.4481 & 0.3309 \\
\hline & 1 & 0.6212 & 0.3700 & 0.2078 & 0.6270 & 0.2587 & 0.1081 & 0.6122 & 0.4742 & 0.3839 \\
\hline & 2 & 0.5929 & 0.2724 & 0.1185 & 0.5703 & 0.1393 & 0.0469 & 0.5802 & 0.4814 & 0.4096 \\
\hline \multirow{3}{*}{$U_{m, k}^{(1)}$} & 0 & 1.2367 & 1.0887 & 1.0000 & 1.2724 & 1.1740 & 1.0000 & 1.0000 & 1.0000 & 1.0000 \\
\hline & 1 & 2.8479 & 1.2782 & 0.7489 & 5.7056 & 1.5562 & 0.6077 & 1.0000 & 1.0000 & 1.0000 \\
\hline & 2 & 5.4083 & 1.3437 & 0.5081 & 13.8442 & 1.5453 & 0.3914 & 1.0000 & 1.0000 & 1.0000 \\
\hline
\end{tabular}

Table 8. AREs of $U_{m, k}^{(2)}$ w.r.t. competing tests for Logistic distribution

\begin{tabular}{|c|c|c|c|c|c|c|c|c|c|c|}
\hline \multirow[b]{3}{*}{ Test } & \multirow[b]{3}{*}{$m$} & \multicolumn{9}{|c|}{$q$ or $(1-q)$} \\
\hline & & \multicolumn{3}{|c|}{0.1} & \multicolumn{3}{|c|}{0.3} & \multicolumn{3}{|c|}{0.5} \\
\hline & & $k=1$ & $k=2$ & $k=3$ & $k=1$ & $k=2$ & $k=3$ & $k=1$ & $k=2$ & $k=3$ \\
\hline \multirow[t]{3}{*}{$S$} & 0 & 1.2367 & 0.8367 & 0.5900 & 1.2724 & 0.7692 & 0.4809 & 1.0000 & 0.7124 & 0.5419 \\
\hline & 1 & 1.1759 & 0.7292 & 0.4179 & 1.0363 & 0.4450 & 0.1901 & 0.9385 & 0.7549 & 0.6251 \\
\hline & 2 & 1.1421 & 0.5398 & 0.2387 & 0.9577 & 0.2405 & 0.0824 & 0.9002 & 0.7673 & 0.6647 \\
\hline \multirow[t]{3}{*}{$M G A$} & 0 & 1.0828 & 0.7326 & 0.5166 & 1.3286 & 0.8032 & 0.5021 & 1.1109 & 0.7914 & 0.6020 \\
\hline & 1 & 1.0296 & 0.6385 & 0.3659 & 1.0821 & 0.4646 & 0.1985 & 1.0426 & 0.8386 & 0.6944 \\
\hline & 2 & 1.0000 & 0.4727 & 0.2090 & 1.0000 & 0.2511 & 0.0860 & 1.0000 & 0.8524 & 0.7384 \\
\hline \multirow[t]{3}{*}{$T_{1}$} & 0 & 1.0000 & 0.6765 & 0.4771 & 1.0000 & 0.6045 & 0.3779 & 1.0000 & 0.7124 & 0.5419 \\
\hline & 1 & 0.9508 & 0.5896 & 0.3379 & 0.8145 & 0.3497 & 0.1494 & 0.9385 & 0.7549 & 0.6251 \\
\hline & 2 & 0.9235 & 0.4365 & 0.1930 & 0.7527 & 0.1890 & 0.0648 & 0.9002 & 0.7673 & 0.6647 \\
\hline \multirow[t]{3}{*}{$T_{2}$} & 0 & 0.7962 & 0.5387 & 0.3799 & 0.9000 & 0.5441 & 0.3401 & 0.7858 & 0.5598 & 0.4258 \\
\hline & 1 & 0.7571 & 0.4695 & 0.2691 & 0.7330 & 0.3147 & 0.1345 & 0.7375 & 0.5932 & 0.4912 \\
\hline & 2 & 0.7353 & 0.3476 & 0.1537 & 0.6774 & 0.1701 & 0.0583 & 0.7074 & 0.6030 & 0.5224 \\
\hline \multirow[t]{3}{*}{$T_{3}$} & 0 & 0.8008 & 0.5418 & 0.3821 & 0.9198 & 0.5561 & 0.3476 & 0.7687 & 0.5476 & 0.4166 \\
\hline & 1 & 0.7614 & 0.4722 & 0.2706 & 0.7492 & 0.3217 & 0.1374 & 0.7215 & 0.5803 & 0.4805 \\
\hline & 2 & 0.7396 & 0.3496 & 0.1546 & 0.6923 & 0.1739 & 0.0596 & 0.6920 & 0.5898 & 0.5110 \\
\hline \multirow{3}{*}{$U_{m, k}^{(1)}$} & 0 & 1.2367 & 1.0887 & 1.0000 & 1.2724 & 1.1740 & 1.0000 & 1.0000 & 1.0000 & 1.0000 \\
\hline & 1 & 2.8479 & 1.2782 & 0.7489 & 5.7056 & 1.5562 & 0.6077 & 1.0000 & 1.0000 & 1.0000 \\
\hline & 2 & 5.4083 & 1.3437 & 0.5081 & 13.8442 & 1.5453 & 0.3914 & 1.0000 & 1.0000 & 1.0000 \\
\hline
\end{tabular}




\section{GOYAL \& KUMAR}

Table 9. AREs of $U_{m, k}^{(2)}$ w.r.t. competing tests for Laplace distribution

\begin{tabular}{|c|c|c|c|c|c|c|c|c|c|c|}
\hline \multirow[b]{3}{*}{ Test } & \multicolumn{10}{|c|}{$q$ or $(1-q)$} \\
\hline & \multirow[b]{2}{*}{$m$} & \multicolumn{3}{|c|}{0.1} & \multicolumn{3}{|c|}{0.3} & \multicolumn{3}{|c|}{0.5} \\
\hline & & $k=1$ & $k=2$ & $k=3$ & $k=1$ & $k=2$ & $k=3$ & $k=1$ & $k=2$ & $k=3$ \\
\hline \multirow[t]{3}{*}{$S$} & 0 & 1.2367 & 0.9571 & 0.7105 & 1.2724 & 0.8718 & 0.5982 & 1.0000 & 0.7407 & 0.5833 \\
\hline & 1 & 1.2634 & 0.8674 & 0.5221 & 1.0490 & 0.4998 & 0.2320 & 0.9341 & 0.7732 & 0.6573 \\
\hline & 2 & 1.2830 & 0.6594 & 0.3054 & 0.9759 & 0.2674 & 0.0986 & 0.8941 & 0.7795 & 0.6897 \\
\hline \multirow[t]{3}{*}{$M G A$} & 0 & 0.9639 & 0.7460 & 0.5538 & 1.3039 & 0.8933 & 0.6130 & 1.1185 & 0.8285 & 0.6524 \\
\hline & 1 & 0.9847 & 0.6760 & 0.4069 & 1.0749 & 0.5122 & 0.2377 & 1.0447 & 0.8648 & 0.7351 \\
\hline & 2 & 1.0000 & 0.5139 & 0.2380 & 1.0000 & 0.2740 & 0.1010 & 1.0000 & 0.8719 & 0.7713 \\
\hline \multirow[t]{3}{*}{$T_{1}$} & 0 & 1.0000 & 0.7739 & 0.5745 & 1.0000 & 0.6851 & 0.4701 & 1.0000 & 0.7407 & 0.5833 \\
\hline & 1 & 1.0216 & 0.7014 & 0.4222 & 0.8244 & 0.3928 & 0.1823 & 0.9341 & 0.7732 & 0.6573 \\
\hline & 2 & 1.0375 & 0.5332 & 0.2470 & 0.7670 & 0.2101 & 0.0775 & 0.8941 & 0.7795 & 0.6897 \\
\hline \multirow[t]{3}{*}{$T_{2}$} & 0 & 0.9816 & 0.7597 & 0.5640 & 1.0731 & 0.7352 & 0.5045 & 0.8216 & 0.6086 & 0.4793 \\
\hline & 1 & 1.0028 & 0.6884 & 0.4144 & 0.8846 & 0.4215 & 0.1956 & 0.7675 & 0.6353 & 0.5401 \\
\hline & 2 & 1.0184 & 0.5234 & 0.2424 & 0.8230 & 0.2255 & 0.0831 & 0.7346 & 0.6405 & 0.5666 \\
\hline \multirow[t]{3}{*}{$T_{3}$} & 0 & 1.1489 & 0.8891 & 0.6601 & 1.1781 & 0.8071 & 0.5539 & 0.8107 & 0.6005 & 0.4729 \\
\hline & 1 & 1.1736 & 0.8058 & 0.4850 & 0.9712 & 0.4628 & 0.2148 & 0.7573 & 0.6268 & 0.5329 \\
\hline & 2 & 1.1919 & 0.6126 & 0.2837 & 0.9036 & 0.2476 & 0.0913 & 0.7249 & 0.6320 & 0.5591 \\
\hline \multirow{3}{*}{$U_{m, k}^{(1)}$} & 0 & 1.2367 & 1.0887 & 1.0000 & 1.2724 & 1.1740 & 1.0000 & 1.0000 & 1.0000 & 1.0000 \\
\hline & 1 & 2.8479 & 1.2782 & 0.7489 & 5.7056 & 1.5562 & 0.6077 & 1.0000 & 1.0000 & 1.0000 \\
\hline & 2 & 5.4083 & 1.3437 & 0.5081 & 13.8442 & 1.5453 & 0.3914 & 1.0000 & 1.0000 & 1.0000 \\
\hline
\end{tabular}

Table 10. AREs of $U_{m, k}^{(2)}$ w.r.t. competing tests for Cauchy distribution

\begin{tabular}{|c|c|c|c|c|c|c|c|c|c|c|}
\hline \multirow[b]{3}{*}{ Test } & \multicolumn{10}{|c|}{$q$ or $(1-q)$} \\
\hline & \multirow[b]{2}{*}{$m$} & \multicolumn{3}{|c|}{0.1} & \multicolumn{3}{|c|}{0.3} & \multicolumn{3}{|c|}{0.5} \\
\hline & & $k=1$ & $k=2$ & $k=3$ & $k=1$ & $k=2$ & $k=3$ & $k=1$ & $k=2$ & $k=3$ \\
\hline \multirow[t]{3}{*}{$S$} & 0 & 1.2367 & 1.1198 & 0.8835 & 1.2725 & 1.0517 & 0.7677 & 1.0000 & 0.9375 & 0.8253 \\
\hline & 1 & 1.3663 & 1.0259 & 0.6473 & 1.1905 & 0.6241 & 0.3002 & 1.0145 & 0.9799 & 0.9117 \\
\hline & 2 & 1.4326 & 0.7818 & 0.3757 & 1.1701 & 0.3404 & 0.1286 & 1.0121 & 0.9897 & 0.9441 \\
\hline \multirow[t]{3}{*}{$M G A$} & 0 & 0.8633 & 0.7817 & 0.6168 & 1.0876 & 0.8989 & 0.6561 & 0.9880 & 0.9262 & 0.8154 \\
\hline & 1 & 0.9537 & 0.7161 & 0.4518 & 1.0175 & 0.5335 & 0.2566 & 1.0023 & 0.9682 & 0.9008 \\
\hline & 2 & 1.0000 & 0.5457 & 0.2623 & 1.0000 & 0.2910 & 0.1099 & 1.0000 & 0.9778 & 0.9328 \\
\hline \multirow[t]{3}{*}{$T_{1}$} & 0 & 1.0000 & 0.9055 & 0.7144 & 1.0000 & 0.8266 & 0.6033 & 1.0000 & 0.9375 & 0.8253 \\
\hline & 1 & 1.1048 & 0.8296 & 0.5234 & 0.9356 & 0.4905 & 0.2359 & 1.0145 & 0.9799 & 0.9117 \\
\hline & 2 & 1.1584 & 0.6321 & 0.3038 & 0.9195 & 0.2676 & 0.1011 & 1.0121 & 0.9897 & 0.9441 \\
\hline \multirow[t]{3}{*}{$T_{2}$} & 0 & 1.2990 & 1.1762 & 0.9280 & 1.3843 & 1.1442 & 0.8351 & 1.2117 & 1.1360 & 1.0000 \\
\hline & 1 & 1.4351 & 1.0776 & 0.6798 & 1.2951 & 0.6790 & 0.3266 & 1.2293 & 1.1874 & 1.1048 \\
\hline & 2 & 1.5047 & 0.8211 & 0.3947 & 1.2729 & 0.3704 & 0.1399 & 1.2264 & 1.1992 & 1.1440 \\
\hline \multirow[t]{3}{*}{$T_{3}$} & 0 & 2.0186 & 1.8278 & 1.4421 & 1.9787 & 1.6354 & 1.1937 & 1.5807 & 1.4819 & 1.3045 \\
\hline & 1 & 2.2301 & 1.6745 & 1.0564 & 1.8511 & 0.9705 & 0.4668 & 1.6037 & 1.5490 & 1.4412 \\
\hline & 2 & 2.3382 & 1.2760 & 0.6133 & 1.8194 & 0.5294 & 0.2000 & 1.5999 & 1.5644 & 1.4924 \\
\hline \multirow{3}{*}{$U_{m, k}^{(1)}$} & 0 & 1.2367 & 1.0887 & 1.0000 & 1.2724 & 1.1740 & 1.0000 & 1.0000 & 1.0000 & 1.0000 \\
\hline & 1 & 2.8479 & 1.2782 & 0.7489 & 5.7056 & 1.5562 & 0.6077 & 1.0000 & 1.0000 & 1.0000 \\
\hline & 2 & 5.4083 & 1.3437 & 0.5081 & 13.8442 & 1.5453 & 0.3914 & 1.0000 & 1.0000 & 1.0000 \\
\hline
\end{tabular}




\section{TESTING OF SCALE PARAMETERS}

From the tables of AREs, one can observe that the performance of the $U_{m, k}^{(1)}$ and $U_{m, k}^{(2)}$ tests depend upon the tail behavior of the underlying distribution. Also, these tests perform better than competing tests for some specified choices of $m$ and $k$. We observe that these tests attain maximum efficiency as follows:

1) For Uniform, Normal, Logistic, and Laplace distributions, $U_{m, k}^{(1)}$ and $U_{m, k}^{(2)}$ tests attain maximum efficiency at $m=0, k=1$ for any $q$. Moreover, for Laplace distribution, $U_{m, k}^{(2)}$ attained maximum efficiency at $m$ as large as possible and $k=1$ for $q \notin(0.3,0.7)$.

2) For the Cauchy distribution, $U_{m, k}^{(1)}$ and $U_{m, k}^{(2)}$ tests attain maximum efficiency at $m=1, k=1$ for $q=0.5$. Now, for $q \neq 0.5$ and $q \in(0.3,0.7)$, $U_{m, k}^{(1)}$ and $U_{m, k}^{(2)}$ attained maximum efficiency at $m=0, k=1$. Moreover, for $q \notin(0.3,0.7), U_{m, k}^{(1)}$ attained maximum efficiency at $m=0, k=2$ and $U_{m, k}^{(2)}$ attained maximum efficiency at $\mathrm{m}$ as large as possible and $k=1$.

3) For the comparison of $U_{m, k}^{(1)}$ and $U_{m, k}^{(2)}$ tests with respect to each other, AREs doesn't depend upon the underlying distribution. In order to attain more efficiency, one should consider $U_{m, k}^{(2)}$ test in comparison to $U_{m, k}^{(1)}$ test.

\section{An Illustrative Example}

To see the execution of the tests $U_{m, k}^{(c)}$, we consider the data of Jung and Parekh (1970), in which the authors worked out a simple method for the determination of the total iron-binding capacity using Hyland Control Sera. They provide the total iron-binding capacity (TIBC) values using the proposed test (abbreviated as JungParekh method) and the Ramsay method for a sample of 20 observations.

Now, it is of interest to check if there is more variation in determination of TIBC by using Jung-Parekh method in comparison to Ramsay method. Both data sets have common quantile of order 0.1, i.e., $q=0.1$. The computed values $U_{m, k}^{(1)}$ and $U_{m, k}^{(2)}$, test statistics along with their $p$-values, are given in Table 11. 


\section{GOYAL \& KUMAR}

Table 11. Computed values of test statistics and corresponding $p$-values

\begin{tabular}{|c|c|c|c|c|c|}
\hline \multirow[b]{2}{*}{$k$} & \multirow[b]{2}{*}{$\boldsymbol{m}$} & \multicolumn{2}{|c|}{$\boldsymbol{U}_{m, k}^{(1)}$} & \multicolumn{2}{|c|}{$\boldsymbol{U}_{m, k}^{(2)}$} \\
\hline & & test statistic & $p$-value & test statistic & $p$-value \\
\hline \multirow[t]{3}{*}{1} & 0 & 0.4000 & 0.4541 & -0.0200 & 0.4490 \\
\hline & 1 & 0.3058 & 0.4311 & -0.0330 & 0.4152 \\
\hline & 2 & 0.2323 & 0.4147 & -0.0330 & 0.4029 \\
\hline \multirow[t]{3}{*}{2} & 0 & 0.2261 & 0.4109 & -0.2730 & 0.4197 \\
\hline & 1 & 0.1521 & 0.3896 & -0.2720 & 0.4099 \\
\hline & 2 & 0.1069 & 0.3760 & -0.2310 & 0.4358 \\
\hline \multirow[t]{3}{*}{3} & 0 & 0.1475 & 0.3988 & -0.3490 & 0.4351 \\
\hline & 1 & 0.0844 & 0.3677 & -0.3440 & 0.4344 \\
\hline & 2 & 0.0529 & 0.3486 & -0.2890 & 0.4699 \\
\hline
\end{tabular}

From the Table 11, it can be seen that both $U_{m, k}^{(1)}$ and $U_{m, k}^{(2)}$ tests statistics don't reject the null hypothesis. Thus, we conclude that in determination of TIBC by using Jung-Parekh method has not more variation in comparison to Ramsay method at $5 \%$ level of significance.

\section{Simulation Study}

We have proposed two test statistics, $U_{m, k}^{(1)}$ and $U_{m, k}^{(2)}$, for testing the hypothesis that the scale parameter is equal to one or greater than one, when distributions have common known quantile $\xi_{q}$ of order $q, 0 \leq q \leq 1$. In order to assess the performance of the proposed test statistics, we compute the asymptotic power of $U_{m, k}^{(1)}$ and $U_{m, k}^{(2)}$ tests by using the Monte Carlo simulation technique. Data is generated from Normal distribution with sample sizes $n_{1}$ and $n_{2}$ as $n_{1}, n_{2}=10,20$, and 30. The scale parameters are considered as $\theta=2,2.5$, and 3 . The number of repetitions carried out is 10,000 and the level of significance is fixed at $5 \%$. The asymptotic power of $U_{m, k}^{(1)}$ and $U_{m, k}^{(2)}$ tests for different values of $m$ and $k$ is given in Tables 12-17. 


\section{TESTING OF SCALE PARAMETERS}

Table 12. Asymptotic power of $U_{m, k}^{(1)}$ for $q=0.1$

\begin{tabular}{|c|c|c|c|c|c|c|c|c|c|c|}
\hline \multirow[b]{2}{*}{$n_{1}, n_{2}$} & \multirow[b]{2}{*}{$\theta$} & \multicolumn{3}{|c|}{$k=1$} & \multicolumn{3}{|c|}{$k=2$} & \multicolumn{3}{|c|}{$k=3$} \\
\hline & & $m=0$ & $m=1$ & $m=2$ & $m=0$ & $m=1$ & $m=2$ & $m=0$ & $m=1$ & $m=2$ \\
\hline \multirow[t]{3}{*}{10,10} & 2.0 & 0.5541 & 0.2896 & 0.2508 & 0.5494 & 0.4080 & 0.3261 & 0.5013 & 0.3907 & 0.3853 \\
\hline & 2.5 & 0.6804 & 0.3203 & 0.2903 & 0.6616 & 0.5124 & 0.4071 & 0.6198 & 0.5070 & 0.4608 \\
\hline & 3.0 & 0.7915 & 0.3953 & 0.3267 & 0.7602 & 0.5714 & 0.4461 & 0.6956 & 0.5529 & 0.5225 \\
\hline \multirow[t]{3}{*}{20,10} & 2.0 & 0.6093 & 0.3615 & 0.3390 & 0.6077 & 0.4940 & 0.3944 & 0.5486 & 0.4695 & 0.4398 \\
\hline & 2.5 & 0.7320 & 0.4052 & 0.3950 & 0.7161 & 0.6091 & 0.4635 & 0.6634 & 0.5734 & 0.5229 \\
\hline & 3.0 & 0.8914 & 0.4910 & 0.4056 & 0.8122 & 0.6796 & 0.4773 & 0.7421 & 0.6312 & 0.5537 \\
\hline \multirow[t]{3}{*}{20,20} & 2.0 & 0.6775 & 0.4562 & 0.4175 & 0.6441 & 0.5602 & 0.4522 & 0.5791 & 0.5385 & 0.4970 \\
\hline & 2.5 & 0.8604 & 0.5145 & 0.4837 & 0.7812 & 0.6728 & 0.5278 & 0.7197 & 0.6358 & 0.5528 \\
\hline & 3.0 & 0.9503 & 0.6235 & 0.5649 & 0.8680 & 0.7654 & 0.5871 & 0.7948 & 0.7101 & 0.6095 \\
\hline \multirow[t]{3}{*}{30,20} & 2.0 & 0.7012 & 0.5309 & 0.4967 & 0.6945 & 0.6199 & 0.5372 & 0.6144 & 0.5972 & 0.5766 \\
\hline & 2.5 & 0.8937 & 0.6209 & 0.5685 & 0.8510 & 0.7380 & 0.5860 & 0.8043 & 0.6994 & 0.6009 \\
\hline & 3.0 & 0.9627 & 0.6874 & 0.6103 & 0.9005 & 0.8489 & 0.6512 & 0.8679 & 0.7972 & 0.6770 \\
\hline \multirow[t]{3}{*}{30,30} & 2.0 & 0.7429 & 0.6283 & 0.5635 & 0.7248 & 0.6784 & 0.6016 & 0.7080 & 0.6556 & 0.6192 \\
\hline & 2.5 & 0.9313 & 0.7010 & 0.6239 & 0.9112 & 0.7820 & 0.6427 & 0.8509 & 0.7421 & 0.6714 \\
\hline & 3.0 & 0.9735 & 0.8017 & 0.7258 & 0.9523 & 0.8965 & 0.7490 & 0.9304 & 0.8506 & 0.7613 \\
\hline
\end{tabular}

Table 13. Asymptotic power of $U_{m, k}^{(1)}$ for $q=0.3$

\begin{tabular}{|c|c|c|c|c|c|c|c|c|c|c|}
\hline \multirow[b]{2}{*}{$n_{1}, n_{2}$} & \multirow[b]{2}{*}{$\theta$} & \multicolumn{3}{|c|}{$k=1$} & \multicolumn{3}{|c|}{$k=2$} & \multicolumn{3}{|c|}{$k=3$} \\
\hline & & $m=0$ & $m=1$ & $m=2$ & $m=0$ & $m=1$ & $m=2$ & $m=0$ & $m=1$ & $m=2$ \\
\hline \multirow[t]{3}{*}{10,10} & 2.0 & 0.3990 & 0.1527 & 0.1075 & 0.3621 & 0.2297 & 0.1606 & 0.3310 & 0.2576 & 0.2217 \\
\hline & 2.5 & 0.5603 & 0.1813 & 0.1327 & 0.4435 & 0.2734 & 0.1942 & 0.4012 & 0.3013 & 0.2574 \\
\hline & 3.0 & 0.7407 & 0.2085 & 0.1624 & 0.5081 & 0.3013 & 0.2028 & 0.4624 & 0.3456 & 0.2885 \\
\hline \multirow[t]{3}{*}{20,10} & 2.0 & 0.4785 & 0.1906 & 0.1218 & 0.4110 & 0.2552 & 0.1914 & 0.3862 & 0.2909 & 0.2603 \\
\hline & 2.5 & 0.6285 & 0.2117 & 0.1730 & 0.5138 & 0.3021 & 0.2196 & 0.4690 & 0.3449 & 0.2892 \\
\hline & 3.0 & 0.8454 & 0.2329 & 0.2011 & 0.5730 & 0.3415 & 0.2375 & 0.5212 & 0.3902 & 0.3510 \\
\hline \multirow[t]{3}{*}{20,20} & 2.0 & 0.6194 & 0.2215 & 0.2119 & 0.5130 & 0.2886 & 0.2318 & 0.4596 & 0.3184 & 0.2984 \\
\hline & 2.5 & 0.8126 & 0.2410 & 0.2250 & 0.6385 & 0.3447 & 0.2592 & 0.5791 & 0.3910 & 0.3379 \\
\hline & 3.0 & 0.9539 & 0.2752 & 0.2568 & 0.7437 & 0.3976 & 0.2864 & 0.6789 & 0.4393 & 0.4090 \\
\hline \multirow[t]{3}{*}{30,20} & 2.0 & 0.6872 & 0.3127 & 0.2910 & 0.5581 & 0.3543 & 0.3205 & 0.4992 & 0.3921 & 0.3646 \\
\hline & 2.5 & 0.8574 & 0.3135 & 0.2890 & 0.6891 & 0.3892 & 0.3610 & 0.6224 & 0.4291 & 0.4113 \\
\hline & 3.0 & 0.9677 & 0.3508 & 0.3137 & 0.7812 & 0.4290 & 0.4035 & 0.7328 & 0.4880 & 0.4398 \\
\hline \multirow[t]{3}{*}{30,30} & 2.0 & 0.7790 & 0.3842 & 0.3217 & 0.6288 & 0.4107 & 0.3516 & 0.5703 & 0.4522 & 0.3997 \\
\hline & 2.5 & 0.9390 & 0.4355 & 0.3668 & 0.7813 & 0.4745 & 0.3958 & 0.7036 & 0.5290 & 0.4620 \\
\hline & 3.0 & 0.9831 & 0.4904 & 0.4105 & 0.8742 & 0.5484 & 0.4697 & 0.8145 & 0.5762 & 0.5004 \\
\hline
\end{tabular}




\section{GOYAL \& KUMAR}

Table 14. Asymptotic power of $U_{m, k}^{(1)}$ for $q=0.5$

\begin{tabular}{|c|c|c|c|c|c|c|c|c|c|c|}
\hline \multirow[b]{2}{*}{$n_{1}, n_{2}$} & \multirow[b]{2}{*}{$\theta$} & \multicolumn{3}{|c|}{$k=1$} & \multicolumn{3}{|c|}{$k=2$} & \multicolumn{3}{|c|}{$k=3$} \\
\hline & & $m=0$ & $m=1$ & $m=2$ & $m=0$ & $m=1$ & $m=2$ & $m=0$ & $m=1$ & $m=2$ \\
\hline \multirow[t]{3}{*}{10,10} & 2.0 & 0.4971 & 0.3110 & 0.2837 & 0.2406 & 0.2538 & 0.2437 & 0.1953 & 0.2308 & 0.2362 \\
\hline & 2.5 & 0.6518 & 0.3891 & 0.3039 & 0.2581 & 0.2764 & 0.2760 & 0.2261 & 0.2533 & 0.2603 \\
\hline & 3.0 & 0.7481 & 0.4709 & 0.3269 & 0.2807 & 0.2987 & 0.3013 & 0.2497 & 0.2691 & 0.2789 \\
\hline \multirow[t]{3}{*}{20,10} & 2.0 & 0.5798 & 0.3866 & 0.3413 & 0.3086 & 0.3225 & 0.3217 & 0.2650 & 0.2699 & 0.2894 \\
\hline & 2.5 & 0.7360 & 0.4898 & 0.3904 & 0.3487 & 0.3573 & 0.3584 & 0.3088 & 0.3192 & 0.3241 \\
\hline & 3.0 & 0.8346 & 0.5733 & 0.4513 & 0.4113 & 0.4276 & 0.4295 & 0.3634 & 0.3708 & 0.4014 \\
\hline \multirow[t]{3}{*}{20,20} & 2.0 & 0.7033 & 0.4662 & 0.3925 & 0.3527 & 0.3719 & 0.3753 & 0.2942 & 0.3116 & 0.3302 \\
\hline & 2.5 & 0.8824 & 0.6124 & 0.4674 & 0.4301 & 0.4495 & 0.4431 & 0.3722 & 0.4096 & 0.4256 \\
\hline & 3.0 & 0.9511 & 0.7083 & 0.6205 & 0.5717 & 0.5861 & 0.5874 & 0.4751 & 0.5289 & 0.5508 \\
\hline \multirow[t]{3}{*}{30,20} & 2.0 & 0.7791 & 0.5966 & 0.4790 & 0.4399 & 0.4613 & 0.4615 & 0.3762 & 0.4046 & 0.4197 \\
\hline & 2.5 & 0.9232 & 0.7603 & 0.5712 & 0.5244 & 0.5393 & 0.5407 & 0.4610 & 0.5014 & 0.5201 \\
\hline & 3.0 & 0.9714 & 0.8562 & 0.7036 & 0.6730 & 0.6916 & 0.6887 & 0.5617 & 0.6209 & 0.6416 \\
\hline \multirow[t]{3}{*}{30,30} & 2.0 & 0.8610 & 0.6376 & 0.5611 & 0.5224 & 0.5396 & 0.5408 & 0.4599 & 0.4928 & 0.5031 \\
\hline & 2.5 & 0.9655 & 0.8028 & 0.6920 & 0.6419 & 0.6622 & 0.6643 & 0.5708 & 0.6227 & 0.6395 \\
\hline & 3.0 & 0.9814 & 0.8931 & 0.8203 & 0.7690 & 0.7784 & 0.7823 & 0.6543 & 0.6796 & 0.6937 \\
\hline
\end{tabular}

Table 15. Asymptotic power of $U_{m, k}^{(2)}$ for $q=0.1$

\begin{tabular}{|c|c|c|c|c|c|c|c|c|c|c|}
\hline \multirow[b]{2}{*}{$n_{1}, n_{2}$} & \multirow[b]{2}{*}{$\theta$} & \multicolumn{3}{|c|}{$k=1$} & \multicolumn{3}{|c|}{$k=2$} & \multicolumn{3}{|c|}{$k=3$} \\
\hline & & $m=0$ & $m=1$ & $m=2$ & $m=0$ & $m=1$ & $m=2$ & $m=0$ & $m=1$ & $m=2$ \\
\hline \multirow[t]{3}{*}{10,10} & 2.0 & 0.6428 & 0.5127 & 0.5092 & 0.6060 & 0.4021 & 0.3195 & 0.3688 & 0.2714 & 0.2018 \\
\hline & 2.5 & 0.6957 & 0.6780 & 0.6612 & 0.6669 & 0.5695 & 0.4914 & 0.5370 & 0.4733 & 0.3923 \\
\hline & 3.0 & 0.8084 & 0.7843 & 0.7719 & 0.7637 & 0.6843 & 0.5623 & 0.6164 & 0.5310 & 0.4809 \\
\hline \multirow[t]{3}{*}{20,10} & 2.0 & 0.6870 & 0.6235 & 0.6114 & 0.6739 & 0.5235 & 0.4476 & 0.4839 & 0.3612 & 0.3247 \\
\hline & 2.5 & 0.8123 & 0.7810 & 0.7634 & 0.7667 & 0.6784 & 0.5850 & 0.6320 & 0.5320 & 0.4538 \\
\hline & 3.0 & 0.8860 & 0.8813 & 0.8635 & 0.8138 & 0.7950 & 0.6324 & 0.6791 & 0.6007 & 0.5617 \\
\hline \multirow[t]{3}{*}{20,20} & 2.0 & 0.7514 & 0.7429 & 0.7297 & 0.7383 & 0.6357 & 0.5680 & 0.5969 & 0.4819 & 0.4202 \\
\hline & 2.5 & 0.9109 & 0.8996 & 0.8689 & 0.8750 & 0.7890 & 0.6973 & 0.7312 & 0.6325 & 0.5460 \\
\hline & 3.0 & 0.9706 & 0.9615 & 0.9562 & 0.9162 & 0.8894 & 0.7912 & 0.8435 & 0.6884 & 0.6457 \\
\hline \multirow[t]{3}{*}{30,20} & 2.0 & 0.8129 & 0.8075 & 0.7782 & 0.7955 & 0.6996 & 0.6142 & 0.6477 & 0.5660 & 0.4814 \\
\hline & 2.5 & 0.9336 & 0.9209 & 0.9028 & 0.8964 & 0.8740 & 0.8180 & 0.8358 & 0.7536 & 0.6691 \\
\hline & 3.0 & 0.9798 & 0.9790 & 0.9693 & 0.9385 & 0.9235 & 0.8562 & 0.8890 & 0.7995 & 0.7170 \\
\hline \multirow[t]{3}{*}{30,30} & 2.0 & 0.8864 & 0.8758 & 0.8640 & 0.8543 & 0.7675 & 0.6795 & 0.7166 & 0.6198 & 0.5709 \\
\hline & 2.5 & 0.9872 & 0.9744 & 0.9609 & 0.9420 & 0.9363 & 0.8879 & 0.9102 & 0.8244 & 0.7482 \\
\hline & 3.0 & 0.9955 & 0.9937 & 0.9882 & 0.9568 & 0.9441 & 0.9118 & 0.9325 & 0.8648 & 0.8195 \\
\hline
\end{tabular}




\section{TESTING OF SCALE PARAMETERS}

Table 16. Asymptotic power of $U_{m, k}^{(2)}$ for $q=0.3$

\begin{tabular}{|c|c|c|c|c|c|c|c|c|c|c|}
\hline \multirow[b]{2}{*}{$n_{1}, n_{2}$} & \multirow[b]{2}{*}{$\theta$} & \multicolumn{3}{|c|}{$k=1$} & \multicolumn{3}{|c|}{$k=2$} & \multicolumn{3}{|c|}{$k=3$} \\
\hline & & $m=0$ & $m=1$ & $m=2$ & $m=0$ & $m=1$ & $m=2$ & $m=0$ & $m=1$ & $m=2$ \\
\hline \multirow[t]{3}{*}{10,10} & 2.0 & 0.5167 & 0.4910 & 0.3206 & 0.3185 & 0.2943 & 0.2722 & 0.3017 & 0.2640 & 0.1638 \\
\hline & 2.5 & 0.6829 & 0.6419 & 0.4383 & 0.4075 & 0.3798 & 0.3509 & 0.3830 & 0.3321 & 0.2544 \\
\hline & 3.0 & 0.7920 & 0.7686 & 0.5202 & 0.5109 & 0.5007 & 0.4812 & 0.5066 & 0.4667 & 0.3792 \\
\hline \multirow[t]{3}{*}{20,10} & 2.0 & 0.5918 & 0.5823 & 0.3916 & 0.3528 & 0.3367 & 0.3106 & 0.3492 & 0.2998 & 0.1875 \\
\hline & 2.5 & 0.7652 & 0.7335 & 0.4955 & 0.4467 & 0.4058 & 0.3845 & 0.4079 & 0.3610 & 0.2867 \\
\hline & 3.0 & 0.8555 & 0.8412 & 0.5867 & 0.5440 & 0.5225 & 0.5026 & 0.5331 & 0.4924 & 0.3833 \\
\hline \multirow[t]{3}{*}{20,20} & 2.0 & 0.7431 & 0.7089 & 0.4368 & 0.3990 & 0.3710 & 0.3373 & 0.3885 & 0.3236 & 0.2493 \\
\hline & 2.5 & 0.9037 & 0.8564 & 0.5712 & 0.5018 & 0.4692 & 0.4384 & 0.4817 & 0.4175 & 0.3328 \\
\hline & 3.0 & 0.9656 & 0.9423 & 0.6598 & 0.6029 & 0.5535 & 0.5244 & 0.5730 & 0.5103 & 0.4390 \\
\hline \multirow[t]{3}{*}{30,20} & 2.0 & 0.8143 & 0.7719 & 0.4918 & 0.4504 & 0.4066 & 0.3680 & 0.4283 & 0.3592 & 0.2609 \\
\hline & 2.5 & 0.9401 & 0.8837 & 0.6509 & 0.5792 & 0.5271 & 0.4966 & 0.5590 & 0.4799 & 0.3814 \\
\hline & 3.0 & 0.9804 & 0.9512 & 0.7183 & 0.6640 & 0.6146 & 0.5797 & 0.6376 & 0.5584 & 0.4725 \\
\hline \multirow[t]{3}{*}{30,30} & 2.0 & 0.8846 & 0.8562 & 0.5367 & 0.4912 & 0.4485 & 0.4002 & 0.4618 & 0.3925 & 0.3017 \\
\hline & 2.5 & 0.9830 & 0.9420 & 0.6795 & 0.6278 & 0.5890 & 0.5330 & 0.5979 & 0.5228 & 0.4439 \\
\hline & 3.0 & 0.9966 & 0.9837 & 0.7795 & 0.7137 & 0.6650 & 0.6243 & 0.6825 & 0.6210 & 0.5523 \\
\hline
\end{tabular}

Table 17. Asymptotic power of $U_{m, k}^{(2)}$ for $q=0.5$

\begin{tabular}{|c|c|c|c|c|c|c|c|c|c|c|}
\hline \multirow[b]{2}{*}{$n_{1}, n_{2}$} & \multirow[b]{2}{*}{$\theta$} & \multicolumn{3}{|c|}{$k=1$} & \multicolumn{3}{|c|}{$k=2$} & \multicolumn{3}{|c|}{$k=3$} \\
\hline & & $m=0$ & $m=1$ & $m=2$ & $m=0$ & $m=1$ & $m=2$ & $m=0$ & $m=1$ & $m=2$ \\
\hline \multirow[t]{3}{*}{10,10} & 2.0 & 0.5028 & 0.3068 & 0.2875 & 0.2515 & 0.2640 & 0.2631 & 0.2023 & 0.2317 & 0.2402 \\
\hline & 2.5 & 0.6579 & 0.3880 & 0.3010 & 0.2691 & 0.2809 & 0.2820 & 0.2297 & 0.2562 & 0.2640 \\
\hline & 3.0 & 0.7715 & 0.4793 & 0.3234 & 0.2898 & 0.3043 & 0.3085 & 0.2512 & 0.2705 & 0.2811 \\
\hline \multirow[t]{3}{*}{20,10} & 2.0 & 0.6169 & 0.3854 & 0.3484 & 0.3101 & 0.3257 & 0.3266 & 0.2697 & 0.2775 & 0.2933 \\
\hline & 2.5 & 0.7684 & 0.4967 & 0.3898 & 0.3523 & 0.3629 & 0.3615 & 0.3113 & 0.3201 & 0.3298 \\
\hline & 3.0 & 0.8890 & 0.6053 & 0.4566 & 0.4184 & 0.4305 & 0.4310 & 0.3692 & 0.3719 & 0.4050 \\
\hline \multirow[t]{3}{*}{20,20} & 2.0 & 0.7332 & 0.4720 & 0.3901 & 0.3569 & 0.3767 & 0.3795 & 0.2978 & 0.3190 & 0.3348 \\
\hline & 2.5 & 0.8935 & 0.6109 & 0.4713 & 0.4387 & 0.4528 & 0.4509 & 0.3824 & 0.4184 & 0.4276 \\
\hline & 3.0 & 0.9676 & 0.7166 & 0.6189 & 0.5758 & 0.5865 & 0.5889 & 0.4770 & 0.5308 & 0.5523 \\
\hline \multirow[t]{3}{*}{30,20} & 2.0 & 0.8125 & 0.5592 & 0.4850 & 0.4413 & 0.4621 & 0.4637 & 0.3780 & 0.4083 & 0.4202 \\
\hline & 2.5 & 0.9413 & 0.7024 & 0.5662 & 0.5275 & 0.5406 & 0.5418 & 0.4639 & 0.5032 & 0.5210 \\
\hline & 3.0 & 0.9819 & 0.8093 & 0.7234 & 0.6743 & 0.6975 & 0.6990 & 0.5698 & 0.6290 & 0.6497 \\
\hline \multirow[t]{3}{*}{30,30} & 2.0 & 0.8740 & 0.6405 & 0.5638 & 0.5277 & 0.5405 & 0.5440 & 0.4674 & 0.4966 & 0.5095 \\
\hline & 2.5 & 0.9725 & 0.8012 & 0.6933 & 0.6480 & 0.6678 & 0.6699 & 0.5725 & 0.6297 & 0.6404 \\
\hline & 3.0 & 0.9911 & 0.8964 & 0.8198 & 0.7703 & 0.7815 & 0.7886 & 0.6584 & 0.6812 & 0.6970 \\
\hline
\end{tabular}




\section{GOYAL \& KUMAR}

Based on the computation of the asymptotic power for Normal distribution, we conclude that:

1) The change in scale of order of 3 is detected for random samples of size $\geq 20$ at $m=0$ and $k=1$ for both $U_{m, k}^{(1)}$ and $U_{m, k}^{(2)}$ tests for any $q$.

2) For all other $U_{m, k}^{(1)}$ and $U_{m, k}^{(2)}$ tests (except at 1 above), a larger sample size is needed to detect the change of scale of the same order.

3) The $U_{m, k}^{(2)}$ test detects the change of scale with more power in comparison to the $U_{m, k}^{(1)}$ test. This validates the computations of AREs as well.

\section{Conclusion}

In this paper, we proposed two classes of distribution-free tests for testing the equality of the scale parameter with common known quantile $\xi_{q}$ of order $q$, $0 \leq q \leq 1$. The proposed classes of tests $U_{m, k}^{(1)}$ and $U_{m, k}^{(2)}$, are generalization of some existing tests. The null distribution of the proposed tests is derived. We compared the proposed class of tests with some existing tests in terms of Pitman asymptotic relative efficiency. It is observed that for some underlying distributions, the proposed tests perform better than competing tests for some choices of $m$ and $k$. We applied the proposed tests on real life data set of Jung and Parekh (1970). The power of the proposed tests is assessed using Monte Carlo simulation study and some conclusions are made for practical implementation.

\section{References}

Ansari, A. R., \& Bradley, R. A. (1960). Rank-sum tests for dispersions. The Annals of Mathematical Statistics, 31(4), 1174-1189. doi:

10.1214/aoms/1177705688

Deshpande, J. V., \& Kusum, K. (1984). A test for the nonparametric two sample scale problem. Australian Journal of Statistics, 26(1), 16-24. doi: 10.1111/j.1467-842X.1984.tb01263.x

Goyal, M., \& Kumar, N. (2018). A generalization of the Sukhatme's test for two-sample scale problem. Journal of Statistical Theory and Applications, 18(2), 155-170. doi: 10.2991/jsta.d.190524.002 


\section{TESTING OF SCALE PARAMETERS}

Jung, D. H., \& Parekh, A. C. (1970). A semi-micromethod for the determination of serum iron and iron-binding capacity without deproteinization. American Journal of Clinical Pathology, 54(6), 813-817. doi: 10.1093/ajcp/54.6.813

Klotz, J. (1962). Nonparametric tests for scale. The Annals of Mathematical Statistics, 33(2), 498-512. doi: 10.1214/aoms/1177704576

Kochar, S. C., \& Gupta, R. P. (1986). Some competitors of the Mood test for the two-sample scale problem. Communications in Statistics - Theory and Methods, 15(1), 231-239. doi: 10.1080/03610928608829117

Kössler, W. (1994). Restrictive adaptive tests for the treatment of the twosample scale problem. Computational Statistics and Data Analysis, 18(5), 513524. doi: 10.1016/0167-9473(94)90081-7

Kössler, W. (1999). Rank tests in the two-sample scale problem with unequal and unknown locations. Statistical Papers, 40(1), 13-35. doi: 10.1007/BF02927108

Kössler, W., \& Kumar, N. (2010). An adaptive test for the two-sample scale problem based on $U$-statistics. Communications in Statistics - Simulation and Computation, 39(9), 1785-1802. doi: 10.1080/03610918.2010.518268

Kössler, W., \& Kumar, N. (2016). An adaptive test for the two-sample scale problem where the common quantile may be different from the median. Statistical Methodology, 29, 10-17. doi: 10.1016/j.stamet.2015.08.001

Kumar, N., \& Goyal, M. (2018). A general class of non parametric tests for comparing scale parameters. Communications in Statistics - Theory and Methods, 47(24), 5956-5972. doi: 10.1080/03610926.2017.1404099

Lehmann, E. L. (1963). Robust estimation in analysis of variance. The Annals of Mathematical Statistics, 34(3), 957-966. doi:

10.1214/aoms/1177704018

Mahajan, K. K., Gaur, A., \& Arora, S. (2011). A nonparametric test for a two-sample scale problem based on subsample medians. Statistics and Probability Letters, 81(8), 983-988. doi: 10.1016/j.spl.2011.01.018

Mood, A. M. (1954). On the asymptotic efficiency of certain nonparametric two-sample tests. The Annals of Mathematical Statistics, 25(3), 514-522. doi: 10.1214/aoms/1177728719

Öztürk, Ö. (2001). A generalization of Ahmad's class of Mann-WhitneyWilcoxon statistics. Australian \& New Zealand Journal of Statistics, 43(1), 67-74. doi: 10.1111/1467-842X.00155 


\section{GOYAL \& KUMAR}

Sukhatme, B.V. (1957). On certain two-sample nonparametric tests for variances. The Annals of Mathematical Statistics, 28(1), 188-194. doi:

10.1214/aoms/1177707046

Tamura, R. (1966). Nonparametric tests for scale. Bulletin of Mathematical Statistics, 12(1/2), 89-94. doi: 10.5109/13020

Yanagawa, T. (1970). A further comparison of two-sample nonparametric tests for dispersion I. Bulletin of Mathematical Statistics, 14(1/2), 15-24. doi:

$10.5109 / 13041$ 